\title{
ON THE NON-VANISHING OF POINCARÉ SERIES
}

\author{
by J. LEHNER
}

(Received 2nd April 1979)

1.

Let $M=S L(2, Z)$ be the classical modular matrix group. One form of the Poincare series on $M$ is

$$
g_{q}(z, m)=\frac{1}{2} m^{2 q-1} \sum_{M_{\infty} \backslash M}(c z+d)^{-2 q} \exp (2 \pi i m A z)
$$

here $z \in \boldsymbol{H}=\{z=x+i y: y>0\}, q \geqq 2$ and $m \geqq 1$ are integers, and the summation is over a complete system of matrices $(a b: c d)$ in $M$ with different lower row. The problem of the identical vanishing of the Poincaré series for different values of $m$ and $q$ goes back to Poincaré.

It is known that $g_{q}$ is a modular form of weight $q$ holomorphic in $\boldsymbol{H}$, i.e.,

$$
g_{q}(A z, m)\left(A^{\prime} z\right)^{q}=g_{q}(z) \forall A \in M .
$$

Since the translation $(11: 01) \in M, g_{q}$ has a Fourier series

$$
g_{q}(z, m)=\sum_{r=1}^{\infty} c_{q}(r, m) \exp (2 \pi i r z),
$$

the summation being over $r \geqq 1$ because of $m \geqq 1$.

From the Petersson scalar product formula, we deduce at once that $g_{q}(z, m) \equiv 0$ if and only if $c_{q}(m, m)=0$. On the other hand there is an explicit formula for $c_{q}$ as an infinite series involving Bessel functions and certain complicated number-theoretic sums (Kloosterman sums).

Very recently $R$. A. Rankin (3) made use of the above to prove the following result.

For each $\varepsilon>0$ there is a $q_{0}(\varepsilon)>0$ such that $g_{q}(z, m) \not \equiv 0$ if

$$
1 \leqq m \leqq q^{2-\varepsilon}, \quad q \geqq q_{0} \text {. }
$$

(Actually Rankin's result was a bit stronger than this.)

In the present note we wish to generalise the above theorem by replacing $M$ by an arbitrary fuchsian group $\Gamma$ that acts on $\boldsymbol{H}$ and has translations. Thus $\Gamma$ may be infinitely generated and it may be of the second kind. However, the inequality we obtain is weaker than (1.4); the exponent is only $\frac{4}{3}$.

We shall prove:

Theorem. Let $\Gamma$ be a fuchsian group acting on $\boldsymbol{H}$ and possessing translations. Let the 
Poincaré series $g_{q}(z, m)$ be defined as in (1.1). Then there exist positive constants $q_{0}$ and $m$, depending only on $\Gamma$, such that $g_{q}(z, m) \neq \equiv$ for.

$$
q \geqq q_{0}, m \leqq m_{12} q^{4 / 3} \text {. }
$$

\section{2.}

Let $\Gamma$ be a fuchsian group acting on $\boldsymbol{H}$ and possessing translations, of which the smallest is $z \rightarrow z+\lambda, \lambda>0$. We represent $\Gamma$ as a group of matrices $G=\{A=(a b: c d), a d-b c=1\}$ with real entries, and we assume without loss of generality that $-I=\left(\begin{array}{lll}-1 & 0: 0 & -1\end{array}\right) \in$ $G$, so that $G / \pm I \cong \Gamma$. Denote by $G_{\infty}=\left\langle\left(\begin{array}{lll}1 \lambda: 0 & 1\end{array}\right)>\right.$ the stabiliser of $\infty$.

The Poincaré series is defined as before by

$$
g_{q}\left(z, m=\frac{1}{2} m^{2 q-1} \sum_{G_{\infty} \backslash G}(c z+d)^{-2 q} \exp (2 \pi i m A z)\right.
$$

with the same conditions on $q$ and $m$ and the same meaning for $G_{\infty} \mid G$. It has the Fourier expansion

$$
g_{q}(z, m)=\sum_{r=1}^{\infty} c_{q}(r, m) \exp (2 \pi i r z / \lambda)
$$

which converges absolutely uniformly on compact subsets of $\boldsymbol{H}$ Before writing down the formula for $c_{q}$ we introduce some further notation $(\mathbf{1}, 270-71,297-98)$.

The set of positive numbers $|c|$ such that $(. .: c.) \in G$ is discrete: $0<c_{0}<c_{1}<\ldots \rightarrow \infty$. For $c=c_{n}>0$ let

$$
D_{c}=\{d:(. . ; c d) \in G, 0 \leqq-d<c \lambda\} ;
$$

this is a finite set. Now define the Kloosterman sum

$$
W_{c}(M, \nu)=\sum_{d \in D_{c}} \exp \left\{2 \pi i \frac{\nu a+m d}{c \lambda}\right\}
$$

where $(a b: c d) \in G_{\infty} \mid G$. Also let

$$
J_{r}(z)=\sum_{n=0}^{\infty} \frac{(-1)^{n}(z / 2)^{r+2 n}}{n ! \Gamma(r+n+1)}
$$

be the Bessel function of the first kind. Then

$$
c_{q}(m, m)=m^{2 q-1}\left\{1+\frac{2 \pi(-1)^{q}}{\lambda} \sum_{n=0}^{\infty} \frac{W_{c_{n}}(m, m)}{c_{n}} J_{2 q-1}\left(\frac{4 \pi m}{c_{n} \lambda}\right)\right\} .
$$

The convergence of (2.2) and formula (2.4) are proved in $(1,276-7,295-8)$ on the assumption that $\Gamma$ is an $\mathrm{H}$-group, but examination of the proof shows this assumption to be unessential.

3.

In showing that $c_{q} \neq 0$ for certain values of $m$, Rankin was able to use very accurate estimates for the Kloosterman sum. But for the general fuchsian group only the trivial 
estimate, obtained by setting each exponential equal to 1 in (2.3), is available. We shall employ the following

Lemma. Let $\sum_{\alpha, \beta}$ denote a sum over

Then

$$
\alpha \leqq c<\beta, \quad 0 \leqq-d<c \lambda
$$

$$
\begin{aligned}
& \sum_{\alpha, \beta} c^{-r}<m_{1} \alpha^{2-r}-m_{2} \beta^{2-r}, \quad r>2 \\
& \sum_{\alpha, \beta} c^{-r}<m_{3} \beta^{2-r}-m_{4} \alpha^{2-r}, \quad r<2
\end{aligned}
$$

where $m_{1}, \ldots$ are positive constants depending only on $\Gamma$.

This result appears as a lemma in (2), p. 400. Again it is proved under the assumption that $\Gamma$ is an $H$-group and again this hypothesis is unnecessary.

Some needed results on Bessel functions can be quoted directly from (3). Write

$$
x_{\nu}=\left(1-\sigma^{4}\right)^{1 / 2}, y_{\nu}=\left(1+\sigma^{4}\right)^{1 / 2}, \quad \sigma=\nu^{-1 / 6} ;
$$

$A_{1}, A_{2}, \ldots$ are absolute constants.

For all $x \geqq 0$ and $\nu \geqq 1$,

$$
\left|J_{\nu}(\nu x)\right| \leqq\left(2 \pi \nu^{-1 / 2}\right)(e x / 2)^{\nu}
$$

This is Lemma 4.1. From Lemma 4.3:

$$
\left|J_{\nu}(\nu x)\right| \leqq A_{2}\left(x^{2}-1\right)^{-1 / 4} \nu^{-1 / 2}, \quad x \geqq y_{\nu} \quad \nu \geqq 15 .
$$

Finally, combining (4.9), (4.10) of (3) we get

$$
\left|J_{\nu}(\nu x)\right| \leqq A_{3} \nu^{-1 / 3}, \quad 2 \geqq x \geqq 2 / e .
$$

Let $q \geqq 8$,

$$
S=\sum_{c>0} \frac{W_{c}(m, m)}{c} J_{2 q-1}(4 \pi(m / c \lambda))
$$

and write

Then

$$
\nu=2 q-1 \geqq 15, \quad Q=4 \pi m / \nu \lambda, \quad x=Q / c \lambda .
$$

$$
\begin{aligned}
|S| \leqq \sum_{c>0} 1 / c\left|J_{\nu}(\nu(Q / c))\right| & \sum_{0 \leqq-d<c \lambda} 1=\sum_{0, \infty} 1 / c\left|J_{\nu}(\nu(Q / c))\right| \\
& =\sum_{0, Q_{1}}+\sum_{O_{1}, Q_{2}}^{\sum_{O_{2}, \infty}}+\sum_{O_{1}}=S_{1}+S_{2}+S_{3},
\end{aligned}
$$

with

$$
Q_{1}=Q / 2 \lambda, \quad Q_{2}=e Q / 2 \lambda
$$


In $S_{3}$ we have $x=Q / c \lambda \geqq 0$, so from (3.3) and (3.1) with $\beta=\infty$, we obtain

$$
\begin{aligned}
\left|S_{3}\right| & \leqq A_{4} \nu^{-1 / 2} \sum_{Q_{2}, \infty} 1 / c\left(\frac{e Q}{2 c \lambda}\right)^{\nu}=A_{4} \nu^{-1 / 2}\left(\frac{e Q}{2 \lambda}\right)^{\nu} \Sigma \frac{1}{c^{\nu+1}} \\
& \leqq m_{5} \nu^{-1 / 2}\left(\frac{e Q}{2 \lambda}\right)^{\nu}\left(\frac{e Q}{2 \lambda}\right)^{1-\nu}=m_{6} \nu^{-1 / 2} Q \\
& =m_{7} m \nu^{-3 / 2}
\end{aligned}
$$

In $S_{1}, x>2>y_{\nu}$, so (3.4) and (3.2) give

$$
\left|S_{1}\right| \leqq A_{2} \nu^{-1 / 2}\left(2^{2}-1\right)^{-1 / 4} \sum_{0, Q_{1}} c^{-1} \leqq m_{8} \nu^{-1 / 2} Q_{1}=m_{9} m \nu^{-3 / 2}
$$

Finally $2 / e<x \leqq 2$ in $S_{2}$; hence by (3.5) and (3.2),

$$
\left|S_{2}\right| \leqq A_{3} \nu^{-1 / 3} \sum_{O_{1}, Q_{2}} c^{-1} \leqq m_{10} \nu^{-1 / 3} \frac{e Q}{2 \lambda}=m_{11} m \nu^{-4 / 3}
$$

It follows that $|S|<\lambda / 2 \pi$ for $q \geqq q_{0}, m \leqq m_{12} q^{4 / 3}$. Then (2.4) shows that

$$
\left|c_{q}(m, m)\right| m^{1-2 q} \geqq 1-\frac{2 \pi}{\lambda}|S|>0
$$

completing the proof of the THEOREM of Section 1.

\section{REFERENCES}

(1) J. LEHNER, Discontinuous groups and automorphic functions (Amer. Math. Soc. Mathematical Surveys no. 8, 1964).

(2) J. LEHNER, On automorphic forms of negative dimension, Illinois J. of Math. 8 (1964), 395-407.

(3) R. A. RAnkin, The vanishing of Poincaré series, Proc. Edinburgh Math. Soc. 23 (1980), 151-161.

DEPARTMENT OF MATHEMATICS

UNIVERSITY OF PITTSBURGH

PITTSBURGH, PENNSYlVANIA

15260, U.S.A. 